\title{
Developing a biomaterial interface based on poly(lactic acid) via plasma-assisted covalent anchorage of D-glucosamine and its potential for tissue regeneration
}

\author{
Ahmed E. Swilem ${ }^{\text {a,b }}$, Marian Lehocký ${ }^{\text {a,* }}$, Petr Humpolíček ${ }^{\text {a }}$, Zdenka Kucekova ${ }^{\text {a }}$, Ita \\ Junkar $^{\mathrm{c}}$, Miran Mozetič ${ }^{\mathrm{c}}$, Ashraf A. Hamed ${ }^{\mathrm{b}}$, Igor Novák ${ }^{\mathrm{d}}$ \\ ${ }^{a}$ Centre of Polymer Systems, Tomas Bata University in Zlín, Trida Tomase Bati 5678, 76001 Zlín, Czech \\ Republic \\ ${ }^{b}$ Department of Chemistry, Faculty of Science, Ain Shams University, Abbassia, Cairo 11566, Egypt \\ ${ }^{c}$ Department of Surface Engineering and Optoelectronics, Jozef Stefan Institute, Jamova Cesta 39, 1000 \\ Ljubljana, Slovenia \\ ${ }^{\mathrm{d}}$ Polymer Institute, Slovak Academy of Sciences, Dúbravská cesta 9, 84541 Bratislava, Slovakia \\ * To whom correspondence should be addressed. E-mail: lehocky@ post.cz, phone: +420 608616048; fax: \\ $+4205766031444$
}

statistical summary: number of words $\mathbf{5 9 7 5}$, number of tables/figures 8

\begin{abstract}
The aim of this study was to develop the potential tissue engineering applications of D-glucosamine $(\mathrm{GlcN})$ immobilized onto the surface of a biodegradable matrix in order to induce a desired biological effect at biointerfaces. Thus, for sample preparation we used a novel multistep physicochemical approach. In the first step the poly(lactic acid) (PLA) films were exposed to a low pressure plasma in air atmosphere, followed by
\end{abstract}


radical graft copolymerization with acrylic acid to yield a carboxyl-functionalized spacer layer on the PLA surface. The carboxyl groups were then coupled to GlcN molecules via the carbodiimide chemistry. The developed surfaces were characterized by X-ray Photoelectron Spectroscopy (XPS), Contact angle measurements and Atomic Force Microscopy (AFM). A preliminary study on the proliferation of fibroblasts on the developed surfaces was performed using the NIH/3T3 cell line.

Keywords: D-glucosamine; Carbodiimide; Poly(lactic acid); Plasma treatment; Fibroblast viability 


\section{Introduction}

Optimization of polymer surface functionalities can control the biological processes such as cell adhesion, proliferation, viability and enhanced extracellular matrix (ECM) secretion functions at biointerfaces [1,2]. Two main strategies in surface modification of polymeric materials are often applied. Firstly, the material surface properties such as chemical composition, wettability, surface charge and roughness, etc. are tailored in such a way that the adsorbed proteins can maintain their normal bioactivities. However, this method cannot induce specific cell behaviors due to the nonspecific protein absorption. The second strategy involves direct immobilization of certain biomolecules onto the biomaterial surfaces in order to induce specific cellular response. Several classes of bioactive molecules, such as proteins, lipids and carbohydrates have been recently utilized for this purpose [3].

Bioabsorbable polymers are considered a suitable alternative to the improvement and development of numerous applications in medicine [4,5]. Poly(lactic acid) (PLA) is one of the most promising biopolymers due to the fact that the monomer "lactic acid" is produced from nontoxic renewable feedstock and it is naturally occurring organic acid as well. The excellent biocompatibility, thermoplastic processability and mechanical properties of PLA widened its use in a variety of biomedical applications. However, as any other synthetic polymer, its surface lacks to bioactive motifs that can be used for specific biorecognition in tissue engineering applications.

Several surface modification techniques have been developed to create new functionalities on polymer surfaces as a precursor for the covalent attachment of bioactive compounds [6]. Among them, plasma treatment is an effective and economical 
surface modification technique for many materials and of growing interests in biomedical engineering [7,8]. Plasma is a gaseous mixture of charged particles, neutral and excited atoms and molecules, radicals and photons. Practically, non-thermal or "cold" plasma which is composed of low temperature particles (charged and neutral molecular and atomic species) and relatively high temperature electrons is convenient for the treatment of heat-sensitive materials such as biodegradable polymers $[9,10]$. Moreover, a unique advantage of plasma modification is that the surface functionalities and characteristics can conveniently be tailored on a nanometer scale without losing desirable physical characteristics of bulk polymer. Plasma can impart the surface with different functionalities and active centers in the form of radicals, peroxides, and hydroperoxides. These active centers can induce a radical graft polymerization in a next step. Finally, the introduced functionalities can be utilized to immobilize relevant biomolecules in order to impart bioactivity to the base polymer.

In particular, carbohydrates are ideal biomolecules for material surface functionalization due to their polyhydroxylated nature. In addition, they are able to convey biological information [11,12]. Carbohydrates are directly implicated in recognition processes including adhesion between cells, adhesion of cells to the extracellular matrix, and specific recognition of cells by one another. Thus, the immobilization of carbohydrates onto polymeric surfaces can be an optimum process for improving simultaneously hydrophilicity, cell adhesion and bioactivity [13-15].

D-Glucosamine $(\mathrm{GlcN})$ is a naturally occurring amino-sugar and it is considered a prominent precursor in the biochemical synthesis of glycosylated proteins and lipids. Therefore, the immobilization of GlcN should be a safe approach for biomaterial surface 
developments. According to the literature, chitosan based materials are relevant candidates in the field of biomaterials, especially for tissue engineering [16,17]. However, the studies on GlcN which represents a contributing monomeric unit in the backbone of chitosan are limited. Recently, GlcN has been used to develop glycosylated polymeric surfaces for different biomedical applications. For example, Russo et al [18] grafted GlcN onto poly( $\varepsilon$-caprolactone) in a single step process of polymer aminolysis for enhancing density and spreading of hMSCs. Wang and Lan [19] developed a glycosylated surface on poly(3-hydroxybutyrate-co-4-hydroxybutyrate) membrane by the chemical attachment of GlcN through a multi-step technique for the selective adsorption of low density lipoprotein.

From the previous summary, it seems that the studies on the cell interactions with GlcN functionalized PLA surfaces prepared by the plasma irradiation route are limited. In the present study, low pressure air plasma was employed to introduce active centers to the PLA film surface. In a subsequent step, such active sites were exploited to initiate the grafting reaction of AAc onto the PLA surface. Lastly, GlcN as a bioactive molecule was chemically immobilized onto the functionalized PLA surface with the aid of 1-ethyl-3-(3dimethylaminopropyl) carbodiimide (EDC) as a condensing agent. Characterization of the developed PLA surfaces was carried out including chemical structure, wettability, surface energy, and morphology. Finally, adhesion and proliferation of NIH/3T3 mouse embryonic fibroblast cells on the untreated and modified PLA films were investigated in order to verify the applicability of these biomaterials in regenerative medicine. 


\section{Materials and methods}

\subsection{Materials}

The commercial PLA 4032D, a semi-crystalline grade with the density of 1.24 $\mathrm{g} / \mathrm{cm}^{3}$ and containing around 2\% D-LA, was purchased in pellets from Nature Works (Blair, NE). Pellets were dried at $60{ }^{\circ} \mathrm{C}$ overnight and then hot-pressed at $180{ }^{\circ} \mathrm{C}$ into films with a thickness of $\sim 150 \mu \mathrm{m}$, followed by cooling in a press.

Acrylic acid (AAc, $99 \%$ ) and 1-ethyl-3-(3-dimethylaminopropyl) carbodiimide hydrochloride (EDC, 98\%) were obtained from Fluka. D-Glucosamine hydrochloride (GlcN, 99\%, suitable for cell culture), ethylene glycol (99.8\%), diiodomethane (99\%) and sodium metabisulfite (99\%) were supplied by Sigma-Aldrich. All reagents were used as received without further purification.

\subsection{Plasma treatment}

Prior to the surface modification by gaseous plasma, the PLA films were cut into rectangles of $4 \mathrm{~cm} \times 5 \mathrm{~cm}$ and rinsed with a detergent solution, then with deionized water several times, and finally dried at room temperature for 2 hours. PLA films were treated from the both sides under a pressure of $60 \mathrm{~Pa}$ in air atmosphere for $120 \mathrm{~s}$ with Pico plasma system (Diener, Germany) operating at $40 \mathrm{kHz}$. The duration of the plasma treatment was optimized by previous experiments by means of water contact angle measurement when equilibrium value was reached for $120 \mathrm{~s}$ and prolonged treatment did not show any contact angle value difference. The power and the air feed rate were set to $50 \mathrm{~W}$ and $20 \mathrm{sccm}$ in all the experiments.

\subsection{Grafting}


Immediately after the plasma treatment, the PLA films were immersed into $10 \mathrm{vol}$ $\%$ aqueous solution of AAc for $24 \mathrm{~h}$ at $30{ }^{\circ} \mathrm{C}$ in order to achieve a radical graft polymerization of AAc. The solution contained $0.1 \mathrm{wt} \%$ of sodium metabisulfite to inhibit AAc homopolymerization. The AAc graft copolymerization led to the creation of poly(acrylic acid) brushes that are suitable for the immobilization of bioactive agents. After that, the AAc- $g$-PLA films were immersed in deionized water for several hours at room temperature and then washed with a copious amount of water several times to remove any unreacted acrylic acid monomer and poly(acrylic acid) homopolymer. The graft density $\left(\mu \mathrm{g} / \mathrm{cm}^{2}\right)$ was determined from Equation (1):

$$
\text { Graft density }=\left(W_{\mathrm{g}}-W_{\mathrm{o}}\right) / S
$$

where $W_{\mathrm{o}}$ and $W_{\mathrm{g}}$ are the weights of PLA film sample before and after grafting and $S$ is the surface area of the PLA sample.

It is important when carrying out the grafting reaction to keep the ratio between the surface area of the PLA film and the volume of the monomer solution fixed. The PLA surface contains the initiating sites for polymerization and, subsequently, its area is considered a determining factor in the grafting reaction.

\subsection{Chemical immobilization of GlcN}

AAc grafted PLA sheets were immersed into $0.1 \mathrm{wt} \%$ aqueous solution of EDC at $4{ }^{\circ} \mathrm{C}$ for $6 \mathrm{~h}$ for the activation of carboxyl groups. EDC reacts with carboxylic acid groups to form an active O-acylisourea intermediate that is easily displaced by nucleophilic attack from primary amino groups in the reaction mixture [20]. The activated carboxyl-functionalized PLA samples were then immersed into 1 wt $\%$ GlcN aqueous solution for $24 \mathrm{~h}$ at $25^{\circ} \mathrm{C}$ in order to allow the amide bond formation. Finally, 
the samples were removed, thoroughly washed with deionized water, and dried for $24 \mathrm{~h}$ at room temperature. The scheme of the multistep approach is given in Fig. 1.

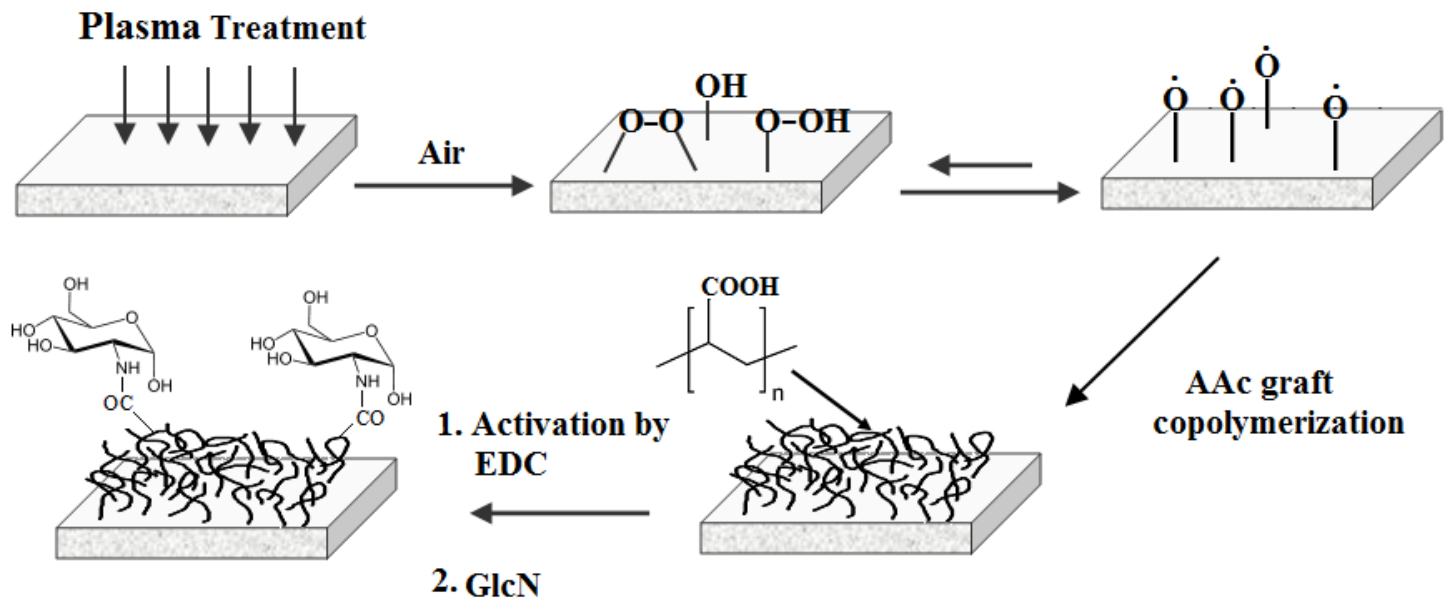

Fig. 1. Schematic diagram for the plasma-assisted immobilization of GlcN onto PLA surface.

\subsection{X-ray photoelectron spectroscopy (XPS)}

X-ray Photoelectron Spectroscopy (XPS) was conducted using TFA XPS Physical Electronics. The base pressure in the XPS analysis chamber was $\approx 6 \times 10^{-8} \mathrm{~Pa}$. The samples were excited by X-rays over a $400-\mu \mathrm{m}$ diameter spot area with a monochromatic $\mathrm{Al} \mathrm{K}_{\alpha 1,2}$ radiation at $1486.6 \mathrm{eV}$. The emitted photoelectrons were detected by a hemispherical analyzer positioned at a take-off angle of $45^{\circ}$. Survey-scan spectra were obtained at a pass energy of 187.85 and $0.4 \mathrm{eV}$ step resolution. An electron gun was employed for surface neutralization. The elemental concentration analysis was performed over three different positions by MultiPak v7.3.1 software. 
Samples were dried at $50{ }^{\circ} \mathrm{C}$ overnight before the analysis to remove any adsorbed gases on the surface. The surface composition for each sample was measured at three different spots allowing the calculation of an average surface composition.

\subsection{Contact angle measurement and surface energy evaluation}

The contact angle data were obtained by the Surface Energy Evaluation System (SEE System; Advex Instruments, Czech Republic). Deionized water, ethylene glycol and diiodomethane were used as the testing liquids. The droplets volume was set to $5 \mu \mathrm{L}$ for all experiments to avoid errors connected with the gravity acting to the sessile drop. All of the calculations were made by the SEE System software for analysis. Six contact angle readings were averaged to obtain one representative value. The substrate surface free energy was evaluated using these data by Lifshitz-Van der Waals "acid-base" model. Prepared samples were measured immediately after preparation.

\subsection{Atomic force microscopy (AFM)}

Surface morphology and roughness of the pristine and treated PLA samples were examined by the AFM technique using the PeakForce TUNA mode on Dimension ICON (Bruker, USA). A silicon tip on nitride lever (ScanAsyst-Air probe; Bruker, USA) with a spring constant of $0.4 \mathrm{~N} \cdot \mathrm{m}^{-1}$ was used. Measurements were conducted at a scanning rate of $0.630 \mathrm{~Hz}$ and a resolution of 512 lines. Three different areas of $5 \mu \mathrm{m}^{2}$ were scanned. The surface roughness was determined using both the arithmetic average of the absolute values of surface height deviations measured from the mean plane $\left(R_{a}\right)$ and the image surface area difference, which is the difference between the images of a three- 
dimensional surface area and a two-dimensional projected surface area. The results were analyzed using NanoScope analysis 9.1 software.

\subsection{Cell proliferation and morphology}

Prior to in-vitro testing, the samples were disinfected by $30 \mathrm{~min}$ of exposure to a UV-radiation source operating at a wavelength of $258 \mathrm{~nm}$ emitted from a low-pressure mercury lamp. Cytotoxicity testing was performed using mouse embryonic fibroblast (ATCC CRL-1658 ${ }^{\mathrm{TM}}$ NIH/3T3) cell line. The ATCC-formulated Dulbecco's Modified Eagle's Medium (BioSera, France) containing 10\% of calf serum (BioSera, France) and $100 \mathrm{U} \mathrm{mL}^{-1}$ Penicillin/Streptomycin (GE Healthcare HyClone, United Kingdom), was used as the culture medium.

Cells were seeded onto square samples $(20 \times 20 \mathrm{~mm})$ in concentration $4 \times 10^{5}$ cells per $\mathrm{mL}$ in volume $500 \mu \mathrm{L}$. After 1 hour, $1500 \mu \mathrm{L}$ of culture medium was added to obtain the final concentration $1 \times 10^{5}$ cells per $\mathrm{mL}$. As a reference, tissue polystyrene was used. To assess cytotoxic effect, the MTT assay (Invitrogen Corporation, USA) was performed after $24 \mathrm{~h}$ of cell cultivation on the samples. All the tests were performed in quadruplicates. The absorbance was measured at $570 \mathrm{~nm}$ by Infinite M200 Pro NanoQuant (Tecan, Switzerland). Dixon's Q test was used to remove outlying values and mean values were calculated. The cell viability is presented as percentage of cells present on the sample surface relatively to the reference ( $100 \%$ viability).

To show the changes in cytoskeleton the DNA staining with Hoechst 33258 (Invitrogen, USA) and actin filaments staining ActinRed ${ }^{\mathrm{TM}} 555$ (Thermo Fisher Scientific, USA) was used. Before staining, cells were fixed and permeabilized. Cells 
were fixed using 4\% formaldehyde (Penta, Czech Republic) for 15 minutes, washed by PBS and subsequently poured with $0.5 \%$ Triton X-100 (Sigma-Aldrich, USA) for 5 minutes to permeabilization. After this time cells were washed 3 times by PBS (Invitrogen, USA). Required amount of PBS, two drops per $1 \mathrm{~mL}$ of ActinRed ${ }^{\mathrm{TM}} 555$ and $5 \mu \mathrm{g} \mathrm{mL}^{-1}$ of Hoechst 33258 were added and left incubate for 30 minutes in the dark. Morphology of cells was observed and micrographs were taken using an inverted Olympus phase contrast microscope (IX 81, Japan).

\section{Results and discussion}

The reactive centers created by plasma treatment in the form of radicals are used immediately to initiate the grafting of AAc onto the PLA surface. The average degree of AAc grafting onto the PLA surface was found to be $\sim 11 \mu \mathrm{g} / \mathrm{cm}^{2}$, and no noticeable weight change was detected after the plasma treatment. From this value of grafting degree, if the grafted chains are confined to the surface only, the grafted layer should be close to $101 \mathrm{~nm}$ in thickness (considering the density of poly(acrylic acid) to be 1.09 $\left.\mathrm{g} / \mathrm{cm}^{3}\right)$. The PLA biointerface was synthesized by conjugating the carboxylic and amino group of GlcN using EDC as a condensing agent. EDC is the most commonly used carbodiimide, since it is soluble in water and is non toxic. Moreover, it is considered a zero-length crosslinker; it causes direct conjugation of carboxyl groups $(-\mathrm{COOH})$ to primary amines $\left(-\mathrm{NH}_{2}\right)$ without adding additional spacer arm atoms.

\subsection{Chemical structure of the developed PLA surfaces}

To confirm the structural changes upon the surface modifications, the XPS technique was used to investigate the prepared surfaces. As shown in Table 1, the XPS 
surface quantitative analysis of the investigated PLA samples clearly ascertains the existing functionalities in the surface region and show subsequent changes in species introduced by each treatment. It can be observed that the measured oxygen-to-carbon $(\mathrm{O} / \mathrm{C})$ ratio of the PLA surface increased from 0.41 to 0.62 after plasma in air atmosphere as a result of the incorporation of oxygen-containing functional groups such as $\mathrm{C}=\mathrm{O}$, $\mathrm{OH}, \mathrm{COOH}, \mathrm{COO}-$, and $\mathrm{C}-\mathrm{O}-\mathrm{C}$ into the surface. Following AAc graft polymerization, the $\mathrm{O} / \mathrm{C}$ ratio remained similar at 0.59 . This ratio is quite close to the theoretical value for the pure poly(acrylic acid) (0.66). This suggests that the grafted layer is well distributed at the surface within the penetration depth of XPS. The biomolecule immobilization step was associated with a reduction in the $\mathrm{O} / \mathrm{C}$ ratio $(0.49)$ and appearance of an N1s signal at $400 \mathrm{eV}$, characteristic for an amide bond. The atomic concentration of N1s was found to be $1.8 \%$. The appearance of N1s peak only for the GlcN modified sample indicates the successful immobilization of this biomolecule onto the PLA surface.

Table 1. XPS surface quantitative analysis and representative high-resolution C1s peak fit data of the investigated PLA samples. (Numbers inside parentheses represent binding energy in eV)

\begin{tabular}{lcccc|ccc}
\hline \multirow{2}{*}{ Sample } & \multicolumn{3}{c}{ Elemental composition (atomic \%) } & \multicolumn{3}{c}{ C1s curve-fitting data (relative area \%) } \\
\cline { 2 - 8 } & $\mathbf{C 1 s}$ & $\mathbf{O 1 s}$ & $\mathbf{N 1 s}$ & $\mathbf{O} / \mathbf{C}$ & $\mathbf{C}-\mathbf{H}$ & $\mathbf{C}-\mathbf{O}(\mathbf{C}-\mathbf{N})$ & $\mathbf{O}=\mathbf{C}-\mathbf{O}$ \\
\hline Untreated PLA & 70.8 & 29.2 & $/$ & 0.41 & $42.8(284.80)$ & $28.6(286.82)$ & $28.6(288.93)$ \\
Plasma treated PLA & 61.6 & 38.4 & $/$ & 0.62 & $33.3(284.84)$ & $32.3(286.80)$ & $34.4(288.95)$ \\
AAc grafted PLA & 62.8 & 37.2 & $/$ & 0.59 & $41.6(284.89)$ & $27.4(286.88)$ & $31.0(288.94)$ \\
GlcN modified PLA & 65.9 & 32.3 & 1.8 & 0.49 & $47.4(284.72)$ & $29.7(286.74)$ & $22.9(288.91)$ \\
\hline
\end{tabular}


To obtain further insight into the chemical bonds present on the surface of the investigated PLA films, a curve-fitting procedure for the high-resolution C1s peaks was performed. The percentage contributions arising from the chemically different $\mathrm{C}$ atoms were calculated from the C1s core level spectrum of each sample (Table 1). The C1s spectrum for the untreated PLA contains three distinct peaks at 284.80, 286.82 and $288.93 \mathrm{eV}$ that can be assigned to carbon atoms of saturated hydrocarbons $(\mathrm{C}-\mathrm{H})$, carbon atoms with a single bond to oxygen $(\mathrm{O}-\underline{\mathrm{C}}-\mathrm{C}=\mathrm{O})$ and carbonyl carbon atoms in the ester group $(\mathrm{O}=\mathrm{C}-\mathrm{O})$, respectively $[21,22]$. After air plasma treatment, the intensity of the hydrocarbon peak decreased while the intensity of $\mathrm{C}-\mathrm{O}$ and $\mathrm{O}=\mathrm{C}-\mathrm{O}$ peaks increased. As a result, it can be concluded that the air plasma mainly attacks the $\mathrm{C}-\mathrm{H}$ bonds in the PLA polymer chains to form $\mathrm{C}-\mathrm{O}$ and $\mathrm{O}=\mathrm{C}-\mathrm{O}$ bonds. The AAc graft copolymerization resulted in an increase in the intensity of the hydrocarbon peak as expected from the relatively high content of saturated hydrocarbons in poly(acrylic acid) $(\sim 67 \%)$ if compared with that in PLA $(\sim 33 \%)$. Moreover, the $\mathrm{C}-\mathrm{O}$ peak intensity was lower if compared with that of PLA or plasma treated PLA because poly(acrylic acid) does not have carbon atoms with a single bond to oxygen. These data confirm the occurrence of grafting of AAc onto the PLA film surface. The chemical immobilization of GlcN onto the AAc grafted PLA surface was accompanied by a substantial increase in the intensity of the hydrocarbon peak and decrease in the intensity of the $\mathrm{O}=\mathrm{C}-\mathrm{O}$ peak. This might be attributed to the structure of $\mathrm{GlcN}$ and to the fact that some of carboxyl groups have reacted with amino groups. In addition, a slight increase in the intensity of the peak associated with $\mathrm{C}-\mathrm{O}$ groups was noticed as a result of the $\mathrm{C}-\mathrm{OH}$ contribution from the monosaccharide attachment on the sample surface [18]. 


\subsection{Surface energy and wettability}

The surface energy is a useful parameter that has often been strongly correlated with the cell-biomaterial interfacial interactions [23]. Generally, the surface energy of solids can be calculated by some indirect methods which are based on contact angle measurements. In this regard, the surface energy of the PLA films was calculated using Van Oss-Chaudhury-Good method. This approach enables to determine the electronacceptor/electron donor "or acid/base" interactions as a component of the surface free energy. The total surface free energy $\left(\gamma^{\text {tot }}\right)$ is a sum of its disperse $L W$ and acid/base $A B$ components, Equation (2):

$$
\gamma^{t o t}=\gamma^{L W}+\gamma^{A B}
$$

where $L W$ indicates the total dispersive Lifshitz-Van der Waals interaction and $A B$ refers to the acid/base interaction. The polar component $\left(\gamma^{A B}\right)$ is further divided into acid and base components according to Equation (3):

$$
\gamma^{A B}=2\left(\gamma^{+} \cdot \gamma^{-}\right)^{1 / 2}
$$

where $\gamma^{+}$and $\gamma^{-}$represent the ion pair acceptor (Lewis acid) contributions and the ion pair donor (Lewis base), respectively.

Table 2 shows the contact angles and surface energy components for the PLA films using deionized water, ethylene glycol and diiodomethane as wetting liquids. Air plasma treatment of the PLA surface caused a significant decrease of the water contact angle and a slight increase of its total surface energy $\left(\gamma^{\text {tot }}\right)$ due to an increase in the polar

component $\left(\gamma^{A B}\right)$. This indicates that plasma treatment improved the hydrophilicity of the PLA surface as a result of the incorporation of characteristic polar functional groups into 
the surface. However, a change in the surface wettability can also be ascribed to many other effects, such as the surface charge or roughness, in addition to alterations in the chemical structure of the film.

Table 2. Contact angle values and surface energy components.

\begin{tabular}{lccc|ccccc}
\hline & \multicolumn{3}{c|}{ Contact angles \pm Standard deviation $\left(^{\circ}\right)$} & \multicolumn{3}{c}{ Surface energy components (mN/m) } \\
\cline { 2 - 9 } Sample & $\boldsymbol{\theta}_{\mathbf{w}}$ & $\boldsymbol{\theta}_{\mathbf{e}}$ & $\boldsymbol{\theta}_{\mathbf{d}}$ & $\gamma^{\text {tot }}$ & $\boldsymbol{\gamma}^{L W}$ & $\gamma^{A B}$ & $\gamma^{+}$ & $\gamma^{-}$ \\
\hline Untreated PLA & $71.8 \pm 3.3$ & $49.2 \pm 1.2$ & $34.9 \pm 2.4$ & 44.59 & 42.06 & 2.53 & 0.11 & 14.45 \\
Plasma treated PLA & $53.0 \pm 2.3$ & $36.9 \pm 1.3$ & $33.6 \pm 1.6$ & 47.53 & 42.68 & 4.85 & 0.17 & 35.04 \\
AA grafted PLA & $61.9 \pm 1.4$ & $37.2 \pm 1.4$ & $30.8 \pm 1.6$ & 44.61 & 43.87 & 0.74 & 0.01 & 20.78 \\
GlcN modified PLA & $60.4 \pm 1.4$ & $32.0 \pm 0.8$ & $29.1 \pm 3.1$ & 45.64 & 44.58 & 1.06 & 0.01 & 20.02 \\
\hline
\end{tabular}

$\theta_{\mathrm{w}}, \theta_{\mathrm{e}}$ and $\theta_{\mathrm{d}}=$ the contact angles for deionized water, ethylene glycol and diiodomethane, respectively.

For standard deviation values the $(n=6)$.

The AAc grafted and GlcN functionalized PLA surfaces showed similar water contact angles which were slightly higher than that for the plasma treated PLA surface but still remained lower than that for the pristine PLA sample. Meanwhile, the surface energy values of those films (44.61and $45.64 \mathrm{mN} / \mathrm{m}$ for AAc grafted and GlcN functionalized PLA, respectively) were slightly lower than that of the plasma treated sample $(47.53 \mathrm{mN} / \mathrm{m})$. This might confirm the chemical functionalization of the PLA surface following the plasma treatment. The polar character of poly(acrylic acid) chains and GlcN moieties is expected to decrease the water contact angle for the PLA surface. However, this also depends on how the polar functional groups oriented themselves on 
the surface as a result of some physical interactions such as hydrogen bonding. Furthermore, the changes in the morphological structures accompanied by the chemical functionalization might affect the water contact angle as well as the surface energy.

It has to be mentioned that the acid component is negligible in all PLA samples if compared with the basic component. This indicates that the tested PLA surfaces have a basic nature. Additionally, the introduction of additional polar groups by the modification processes might be confirmed by an increase in the values of the basic components for all treated samples if compared with that calculated for untreated PLA [24]. Despite the fact that high surface free energies or wettability promote cell adhesion as reported by many researchers, this is not strictly reported as true across the board. This is because wettability and surface free energy are not necessarily directly correlated and because the surface interactions are complex and depend on other factors [25]. However, the untreated and modified PLA surfaces exhibited moderate wettability ranging from $\sim 53$ to $\sim 72^{\circ}$, which is recommended for biomaterials to achieve ideal levels of cell attachment and spreading [26].

\subsection{Surface morphology}

Atomic force microscopy (AFM) is a powerful tool to study the morphological changes and the roughness of the polymer surfaces after plasma and chemical treatments. Fig. 2 shows the 3D AFM images and the roughness parameters at $5 \mu \mathrm{m}$ scale for (a) untreated, (b) plasma treated, (c) AAc grafted, and (d) GlcN modified PLA film surfaces. The surface topography of the untreated PLA had relatively smooth structures randomly distributed across the surface and accompanied by slight defects resulting from the film preparation. Plasma treatment for $120 \mathrm{~s}$ transformed the original smooth surface of PLA 
into a rougher surface filled with nanoscale conical protuberances. This would be most likely due to the etching effect of applied plasma. The interaction of energetic particles such as electrons, ions, radicals, neutrals and excited atoms/molecules as well as UV radiation with the surface of polymer films resulted in etching of the surface [27]. After AAc grafting, the PLA surface morphology appeared different; the nanoscale conical protuberances mostly disappeared and it seems that poly(acrylic acid) brushes were created on the PLA surface after initiating the grafting process by the radicals generated on the film surface. Although the average roughness $\left(\mathrm{R}_{\mathbf{a}}\right)$ for this sample is similar to that of plasma treated one, the surface area difference is substantially decreased. This result is attributed to how the roughness is estimated by the two parameters. In fact, depending on the frequency and distribution of surface projections, these parameters can give very different results for the surface roughness. Generally, an increase in peak count may not significantly affect the average roughness, whereas it can dramatically increase the surface area difference [28]. Therefore, due to the relatively low peak frequency in the AAc grafted sample, if compared with the plasma treated one, the surface area difference is noticeably decreased. This means that the AAc grafting process produced a smoother surface with poly(acrylic acid) brush-like structures whose average of surface height deviations from the mean plane is almost similar to the plasma treated surface. On the final step of modification with GlcN, no further significant changes were noticed in surface morphology, whereas the roughness is further increased which may be due to the biomolecule domains at the surface. 

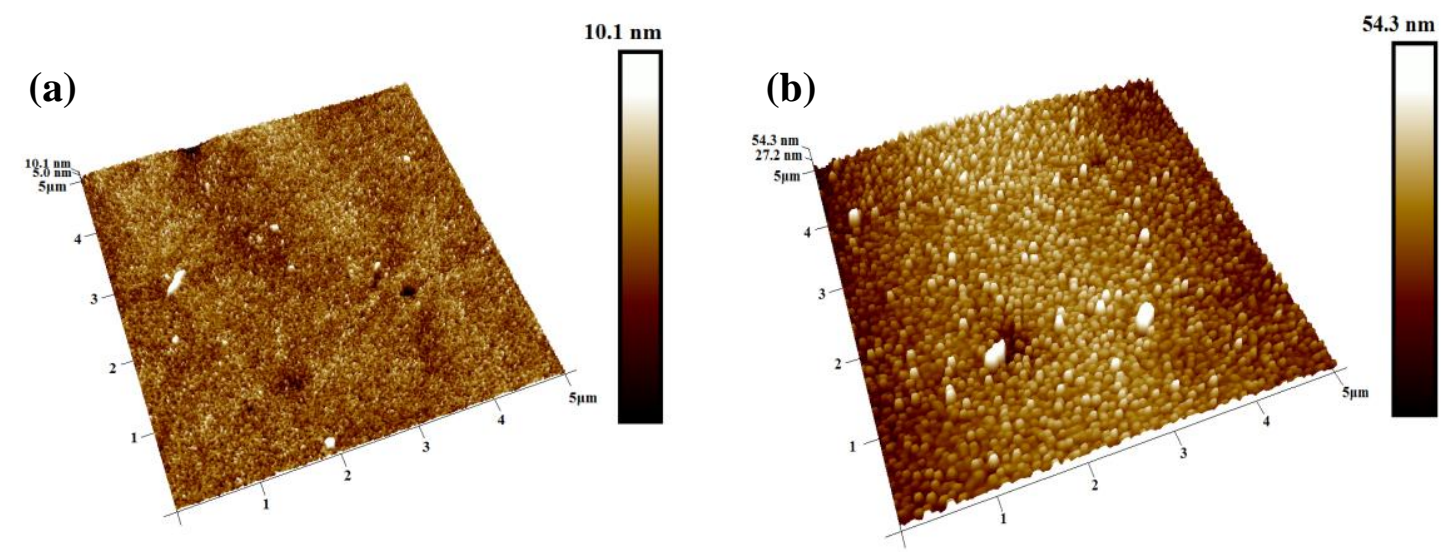

\section{$\mathbf{R}_{\mathrm{a}}: 1.00 \mathrm{~nm}$}

Surface area difference: $1.26 \%$

$R_{\mathrm{a}}: 5.85 \mathrm{~nm}$

Surface area difference: $\mathbf{5 . 1 7} \%$

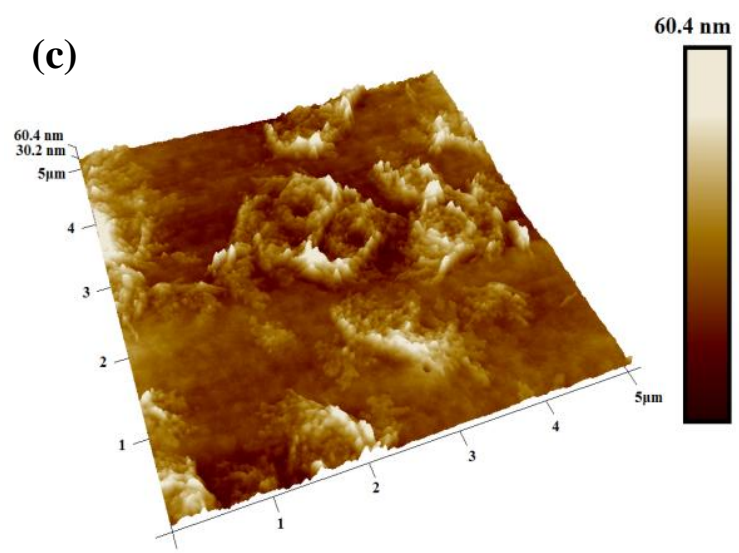

\section{$R_{\mathrm{a}}: 5.95 \mathrm{~nm}$}

Surface area difference: $2.63 \%$

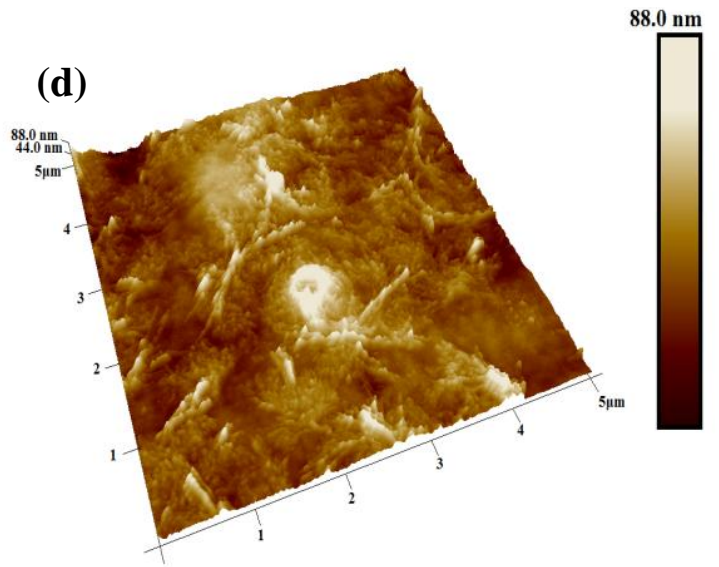

$\mathbf{R}_{\mathrm{a}}: \mathbf{8 . 8 7} \mathrm{nm}$

Surface area difference: $4.90 \%$

Fig. 2. 3D AFM images and roughness parameters for the untreated (a), plasma treated (b), AAc grafted (c), and GlcN modified PLA films (d). (Scan size: $5 \mu \mathrm{m} \times 5 \mu \mathrm{m}$ )

Uverall, these results indicate that the surtace morpnology of the rLA I1Im is

significantly affected by biomolecule immobilization. Moreover, the modified surfaces exhibited increased roughness on nanometer length scales compared to the untreated film. These effects could be favorable for cell adhesion on the PLA surface. The morphology can influence the ability of the substratum to adsorb protein and/or alter the conformation of the adsorbed protein, as cell attachment is always mediated by ECM proteins present in serum used in most cell cultures [23]. 


\subsection{Cell proliferation and morphology}

A study on the viability of living cells on the developed surfaces is essential for the GlcN application in tissue engineering. Therefore, NIH/3T3 fibroblast cells were seeded on the untreated and all modified surfaces to investigate their behaviors.

Biocompatibility can be anticipated from the number of adhered and proliferated cells. Two phases are distinguished in cell culture. In the first phase, adhesion is realized from the first contact between the substrate and the cell surface. Following stage is called lag-phase. In this phase the cells react with the new environment and adapt to it. If the cells survive this phase, they begin to grow, reproduce and proliferate. The cell viability of NIH/3T3 mouse embryonic fibroblast cells on the individual surfaces was evaluated by MTT assay. The results presented in Fig. 3 clearly show that all the treatments improve the ability of cells to adhere and proliferate on the PLA surfaces. All the treatments show viability similar to the reference tissue culture polystyrene. The good cell behavior on the treated PLA samples is also demonstrated by micrographs presented in Fig. 4. Except of the untreated PLA, the cells create confluent population on all the samples without significant changes in their cytoskeleton. 


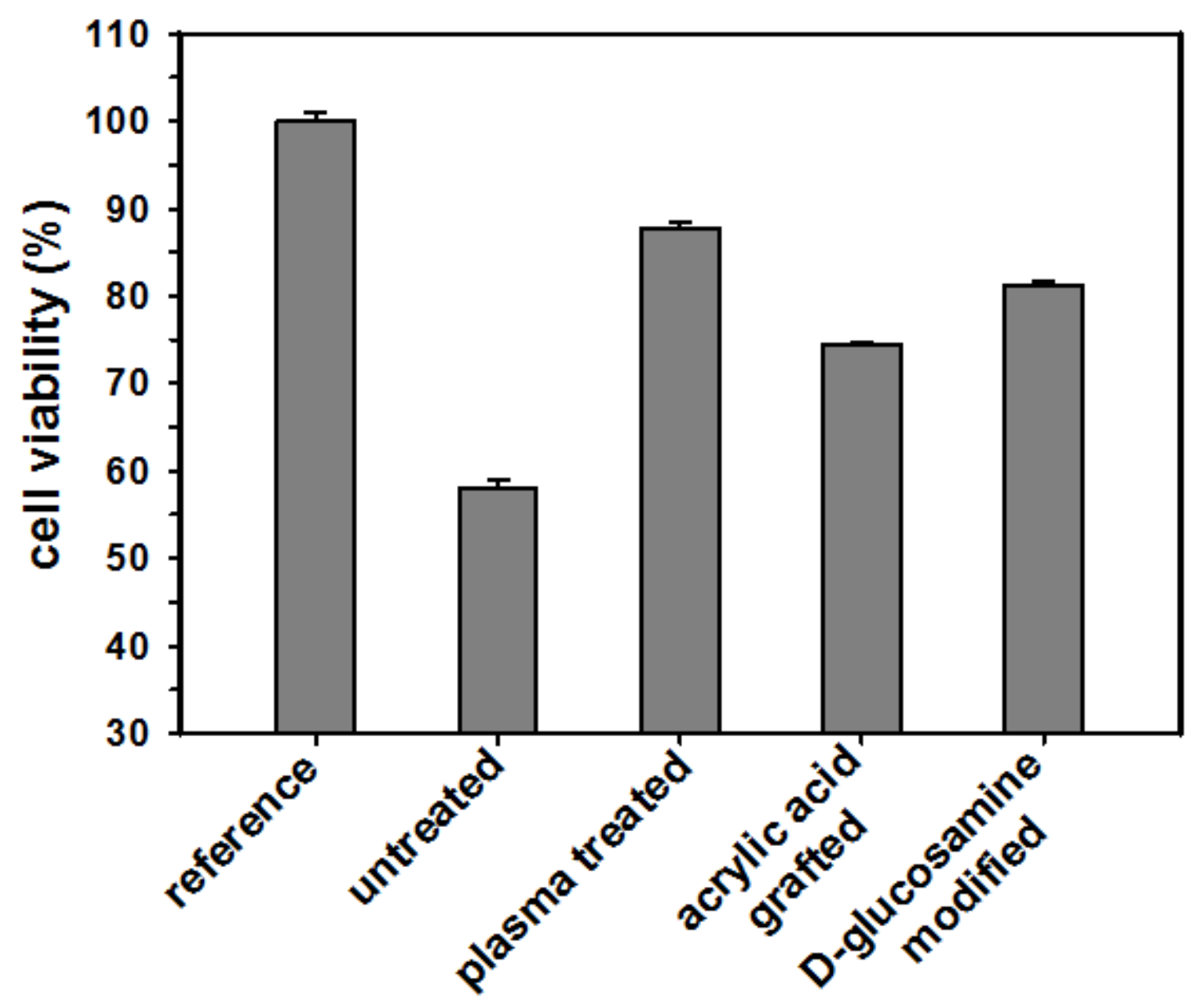

Fig. 3. Cytotoxicity presented as percentage of cell viability cultured on the untreated and modified PLA surfaces compared to the reference. The error bars represent standard deviation $(n=4)$.

At the moment, it is difficult to identify exactly the mechanism by which the cells interacted with each surface. Surface chemistry, roughness, morphology, etc. are key factors in controlling these biological processes. According to the literature, the positive effect of the plasma modification on the cell adhesion and proliferation is induced by a combination of surface properties such as surface roughness, morphology, chemical 
structure or wettability [29]. Although it was reported that the poly(acrylic acid) chains are toxic to smooth muscle cells as might be caused by the acid environment of acrylic acid [30], the results here display a positive effect. This might be attributed to a carboxylprotein coupling reaction [31]. For the GlcN functionalized surface, the biochemical properties as well as the hydroxylated nature of this monosaccharide can account for its biocompatibility [32].
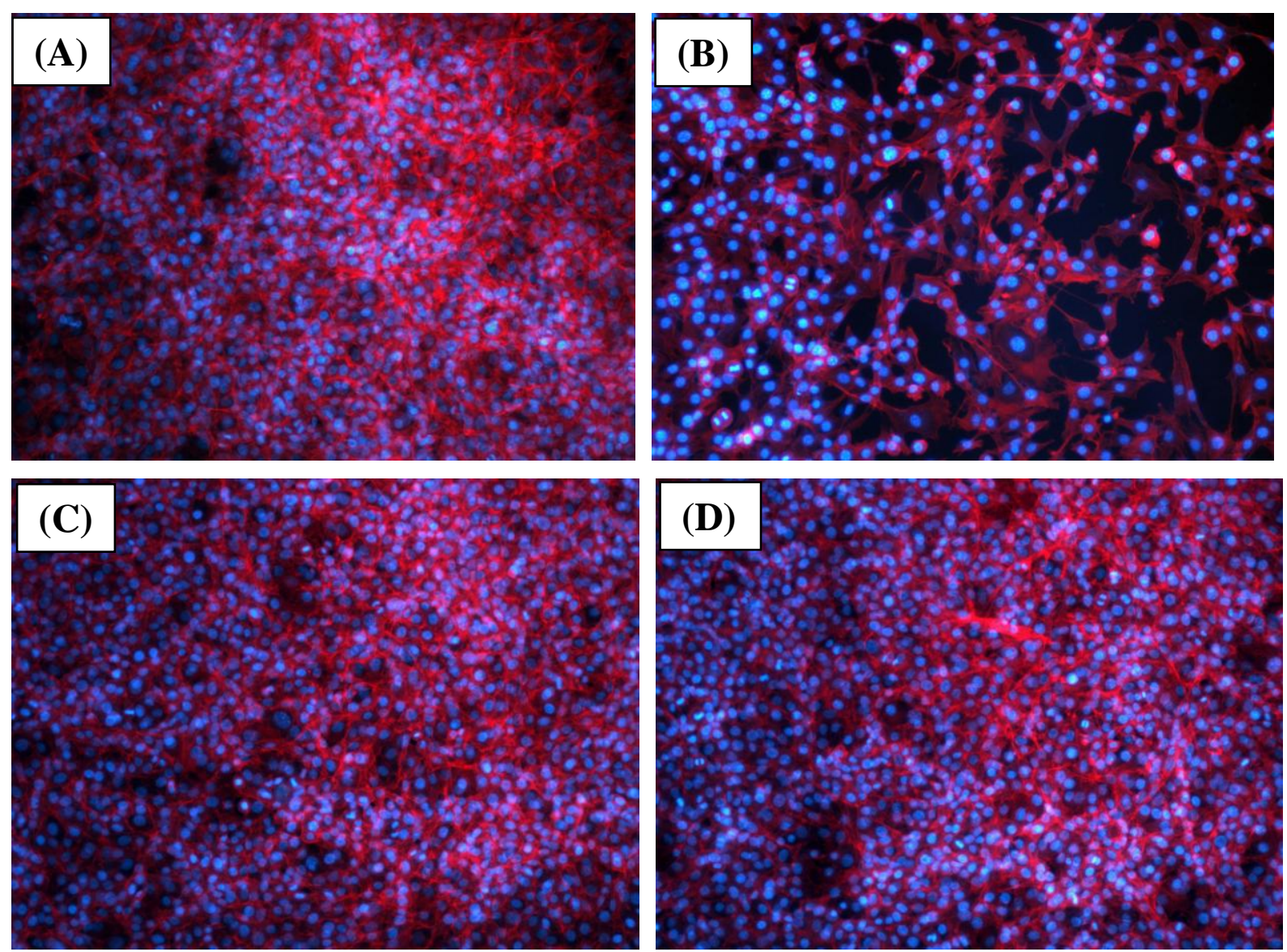

Fig. 4. The cell morphology on A) reference; B) PLA; C) AAc grafted PLA; D) GLcN grafted PLA. Individual cells are visualized through mouse embryonic fibroblast cell nuclei counterstain by Hoechst 33258 (blue colour), the mouse embryonic fibroblast cell cytoskeleton is visualized by ActinRed $^{\mathrm{TM}} 555$ (red colour). 
Nevertheless, it can be concluded that the samples can be considered as biocompatible. In addition, even though the results have demonstrated an enhancement of cell viability for all treated PLA surfaces, GlcN immobilized onto the spacer layer on PLA is more suitable for fibroblasts adhesion and proliferation than the plasma treated surface as demonstrated in Fig 3. This conclusion arises from the fact that plasma treated surfaces are thermodynamically subject to hydrophobic recovery. Therefore, the instability of the plasma treated PLA surface with the increase in aging time limits its application [29, 33, 34].

\section{Conclusion}

The present work showed the effectiveness of plasma treatment to create new functionalities on the PLA surface as a precursor for the covalent attachment of GlcN biomolecule. XPS measurements confirmed the success of the amino-sugar immobilization onto the surface of PLA. The surface modifications of the PLA were associated with noticeable changes in surface wettability and morphology. All treatments caused enhancement of the wettability and increased roughness of the PLA surface. These effects might be favorable for cell viability in tissue engineering applications. The biocompatibility of the untreated and modified PLA surfaces was tested by cell viability assay of NIH/3T3 mouse embryonic fibroblast cells. It was observed that all the treatments improved the ability of cells to adhere and proliferate on the surfaces of PLA and the results are similar to the proliferation on the reference tissue culture polystyrene. Moreover, cell morphology indicated that with the exception of the untreated sample the cells create confluent population without significant changes in their cytoskeleton. 
However, the AAc grafted and GlcN functionalized surfaces is more suitable because the instability of the plasma treated PLA surface with aging limits its application. In conclusion, this study clearly demonstrated the promising potential of GlcN immobilized onto the spacer layer to impart biofunctional activity to PLA in order to enhance the cell adhesion and proliferation in the field of tissue regeneration.

\section{Acknowledgements}

This work was supported by the Ministry of Education, Youth and Sports of the Czech Republic (Project no. LO1504) and the scholarship awarded by virtue of the bilateral cultural agreement between the Czech Republic and the Arab Republic of Egypt and the Ministry of Education, Science, Research and Sport of the Slovak Republic and Slovak Academy of Sciences, project VEGA No. 2/0199/14. The research was partially supported by Slovenian Research Agency, program P2-0082.

\section{References}

[1] Z. Ma, Z. Mao, C. Gao, Surface modification and property analysis of biomedical polymers used for tissue engineering, Colloid Surface B 60 (2007) 137-157.

[2] D.S.W. Benoit, M.P. Schwartz, A.R. Durney, K.S. Anseth, Small functional groups for controlled differentiation of hydrogelencapsulated human mesenchymal stem cells, Nat. Mater. 7 (2008) 816-823.

[3] J. Rodríguez-Hernández, Patterning and functionalization of polymeric Surfaces, in: J. Rodríguez-Hernández, A.L. Cortajarena (Eds.), Design of Polymeric Platforms for 
Selective Biorecognition, Springer International Publishing, Switzerland, 2015, pp. $23-24$.

[4] M.S. Lopes, A.L. Jardini, R.M. Filho, Poly (Lactic Acid) production for tissue engineering applications, Procedia Engineering, Procedia Engineering 42 (2012) 14021413.

[5] K.M. Nampoothiri, N.R. Nair, R.P. John, An overview of the recent developments in polylactide (PLA) research, Biores. Technol. 101 (2010) 8493-8501.

[6] J.M. Goddard, J.H. Hotchkiss, Polymer surface modification for the attachment of bioactive compounds, Prog. Polym. Sci. 32 (2007) 698-725.

[7] P.K. Chu, J.Y. Chen, L.P. Wang, N. Huang, Plasma-surface modification of biomaterials, Mater. Sci. Eng. R-Rep. 36 (2002) 143-206.

[8] F.S. Denes, S. Manolache, Macromolecular plasma-chemistry: an emerging field of polymer science, Prog. Polym. Sci. 29 (2004) 815-885.

[9] A. Bogaerts, E. Neyts, R. Gijbels, J. van der Mullen, Gas discharge plasmas and their applications, Spectrochim. Acta B 57 (2002) 609-658.

[10] M. Laroussi, Low-temperature plasmas for medicine?, IEEE T. Plasma Sci. 37 (2009) 714-725.

[11] P. Stallforth, B. Lepenies, A. Adibekian, P. H. Seeberger, Carbohydrates: A Frontier in Medicinal Chemistry, J. Med. Chem. 52 (2009) 5561-5577.

[12] L. Cipolla, A. C. Araújo, D. Bini, L. Gabrielli, L. Russo, N. Shaikh, Discovery and design of carbohydrate-based therapeutics, Expert Opin. Drug Discovery 5 (2010) 721737. 
[13] L. Cipolla, L. Russo, F. Taraballi, C. Lupo, D. Bini, L. Gabrielli, A. Capitoli, F. Nicotra, Smart biomaterials: the contribution of glycoscience, in, A.P. Rauter, T.K. Lindhorst (Eds.), Carbohydrate Chemistry: Chemical and Biological Approaches, Vol. 38, RSC publishing, Cambridge, 2012, pp. 416-445.

[14] D.B. Werz, Chemical synthesis of carbohydrates and their surface immobilization: a brief introduction, in: Y. Chevolot (Ed.), Carbohydrate Microarrays: Methods and Protocols, Methods in Molecular Biology, vol. 808, Humana Press, New York, 2012, pp. $13-29$.

[15] A. Mateescu, M. Vamvakaki, Glycosurfaces, in: R. Narain (Ed.), Engineered Carbohydrate-Based Materials for Biomedical Applications: Polymers, Surfaces, Dendrimers, Nanoparticles, and Hydrogels, John Wiley \& Sons Inc., Hoboken, 2011, $416-445$.

[16] X. Zhu, K.S. Chian, M.B.E. Chan-Park, S.T. Lee, Effect of argon-plasma treatment on proliferation of human-skin-derived fibroblast on chitosan membrane in vitro, J. Biomed. Mater. Res. A 73 (2005) 264-274.

[17] F. Croisier, C. Jérôme, Chitosan-based biomaterials for tissue engineering, Eur. Polym. J. 49 (2013) 780-792.

[18] L. Russo, A. Gloria, T. Russo, U. D’Amora, F. Taraballi, R. De Santis, L. Ambrosio, F. Nicotra, L. Cipolla, Glucosamine grafting on poly(e-caprolactone): a novel glycated polyester as a substrate for tissue engineering, RSC Adv. 3 (2013) 6286-6289.

[19] W. Wang, P. Lan, Surface glycosylation of poly(3-hydroxybutyrateco-4hydroxybutyrate) membrane for selective adsorption of low-density lipoprotein, $\mathrm{J}$. Biomat. Sci. Polym. E. 25 (2014) 2094-2112. 
[20] N. Nakajima, Y. Ikada, Mechanism of Amide Formation by Carbodiimide for Bioconjugation in Aqueous Media, Bioconjugate Chem. 6 (1995) 123-130.

[21] Y. Zhao, A. Fina, A. Venturello, F. Geobaldo, Effects of gas atmospheres on poly(lactic acid) film in acrylic acid plasma treatment, Appl. Surf. Sci. 283 (2013) 181187.

[22] D. Briggs, Surface Analysis of Polymers by XPS and Static SIMS, first ed., Cambridge University Press, Cambridge, 1998.

[23] A. Nori, E.K.F. Yim, S. Hen, K.W. Leong, Cell substrate interactions, in: A. Atala, R. Lanza, J.A. Thomson, R.M. Nerem (Eds.), Principles of Regenerative Medicine, Academic Press, New York, 2010, pp. 669-670.

[24] P.M. López-Pérez, A.P. Marques, R.M P. da Silva, I. Pashkulevaab, R. L. Reis, Effect of chitosan membrane surface modification via plasma induced polymerization on the adhesion of osteoblast-like cells, J. Mater. Chem. 17 (2007) 4064-4071.

[25] M.M. Gentleman, E. Gentleman, The role of surface free energy in osteoblastbiomaterial interactions, Int. Mater. Rev. 59 (2014) 417-429.

[26] Y. Arima, H. Iwata, Effect of wettability and surface functional groups on protein adsorption and cell adhesion using well-defined mixed self-assembled monolayers, Biomaterials 28 (2007) 3074-3082.

[27] S.K. Pankaj, C. Bueno-Ferrer, N.N. Misra, L. O'Neill, A. Jiménez, P. Bourke, P.J. Cullen, Characterization of polylactic acid films for food packaging as affected by dielectric barrier discharge atmospheric plasma, Innov. Food Sci. Emerg. Technol. 21 (2014) 107-113. 
[28] C. Hobbs, S. Hong, J. Taylor, Effect of surface roughness on fouling of RO and NF membranes during filtration of a high organic surficial groundwater, J. Water Supply Res. T 55 (2006) 7-8.

[29] N. S. Kasálková, P. Slepička, L. Bačáková, P. Sajdl, V. Švorčík, Biocompatibility of plasma nanostructured biopolymers, Nucl. Instr. Meth. Phys. Res. B 307 (2013) 642-646.

[30] B. Gupta, C. Plummer, I. Bisson ,P. Frey, J. Hilborn, Plasma-induced graft polymerization of acrylic acid onto poly(ethylene terephthalate) films: characterization and human smooth muscle cell growth on grafted films, Biomaterials 23 (2002) 863-871. [31] P. Thevenot, W. Hu, L. Tang, Surface chemistry influence implant biocompatibility, Curr. Top. Med. Chem. 8 (2008) 270-280.

[32] S. Varghese, P. Theprungsirikul, S. Sahani, N. Hwang, K. J. Yarema, J. H. Elisseeff, Glucosamine modulates chondrocyte proliferation, matrix synthesis, and gene expression, OsteoArthr. Cartilage 15 (2007) 59-68.

[33] M. Mozetič, G. Primc, A. Vesel, R. Zaplotnik, M. Modic, I. Junkar, N. Recek, M. Klanjšek-Gunde, L. Guhy, M.K. Sunkara, M.C. Assensio, S. Milosevic, M. Lehocký, V. Sedlarik, M. Gorjanc, K. Kutasi, K Stana-Kleinschek, Application of Extremely NonEquilibrium Plasmas in the Processing of Nano and Biomedical Materials, Plasma Sources Science and Technology, 24 (2015) 015026 (12pp).

[34] M. Mozetič, K. Ostrikov, D.N. Ruzic, D. Curreli, U. Cvelbar, A. Vesel, G. Primc, M. Leisch, K. Jousten, O.B. Malyshev, J.H. Hendricks, L. Kövér, A. Tagliaferro, O. Conde, J.A. Silvestre, J. Giapintzakis, M. Buljan, N. Radic, G. Drazic, S. Bernstorff., H. Biederman, O. Kylián, J. Hanuš, S. Miloševič, A. Galtayries, P. Dietrich, W. Unger, M. Lehocký, V. Sedlarik, K. Stana-Kleinschek, A. Drmota-Petrič, J.J. Pireaux, J.W. Rogers, 
M. Anderle, Recent Advances in Vacuum Sciences and Applications, Journal of Physics D: Applied Physics, 47 (2014) 153001 (23pp). 
Click here to download high resolution image

\section{Plasma Treatment}
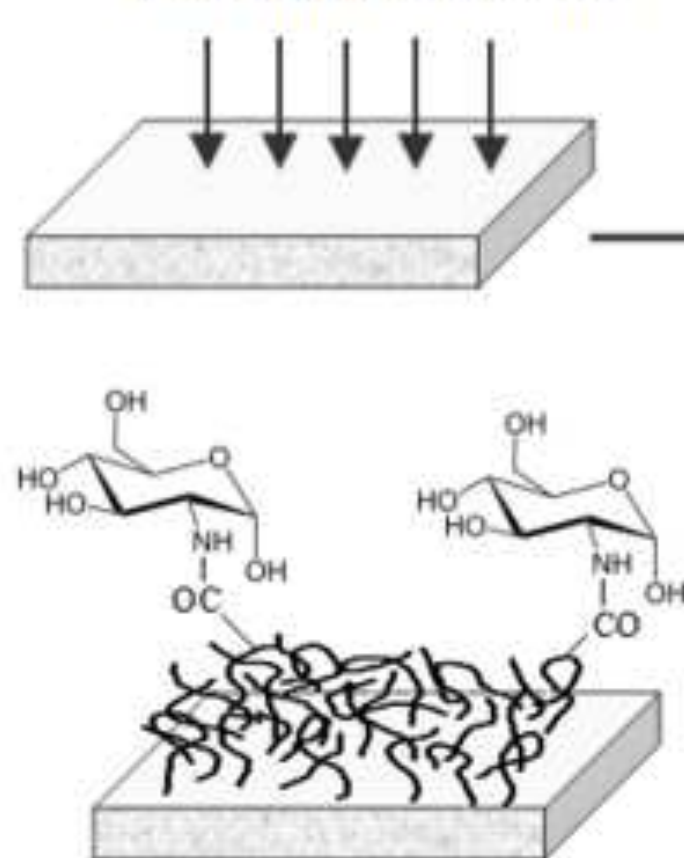

1. Activation by EDC
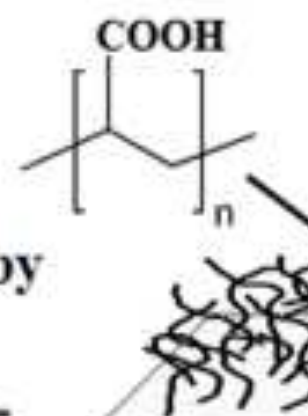
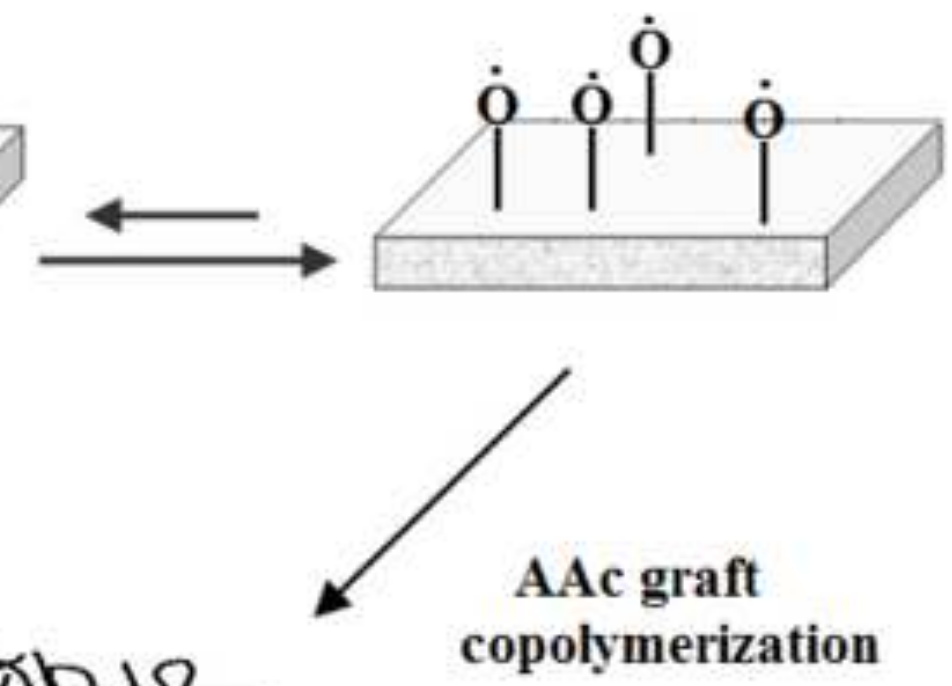

\section{GIcN}


Click here to download high resolution image
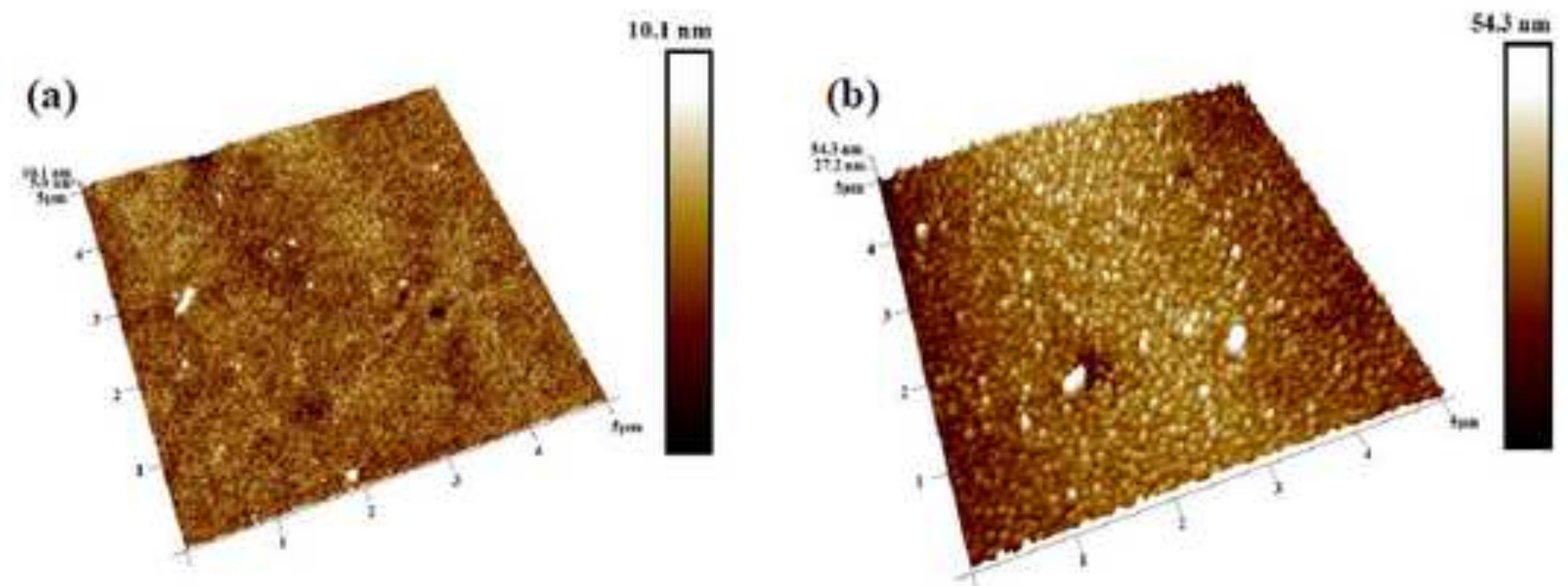

$\mathrm{R}_{\mathrm{a}}: 1.00 \mathrm{~nm}$

Surface area difference: $1.26 \%$

$$
\begin{aligned}
& \mathbf{R}_{\mathrm{a}}: \mathbf{5 . 8 5} \mathrm{nm} \\
& \text { Surface area difference: } 5.17 \%
\end{aligned}
$$

(c)

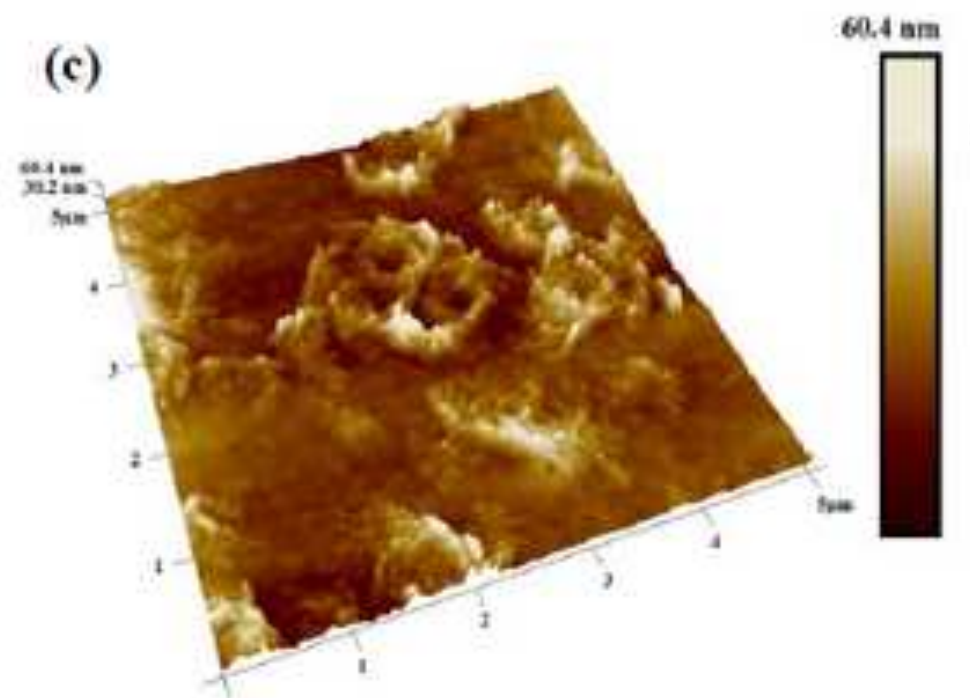

$\mathrm{R}_{\mathrm{n}}: 5.95 \mathrm{~nm}$

Surface area difference: $\mathbf{2 . 6 3} \%$

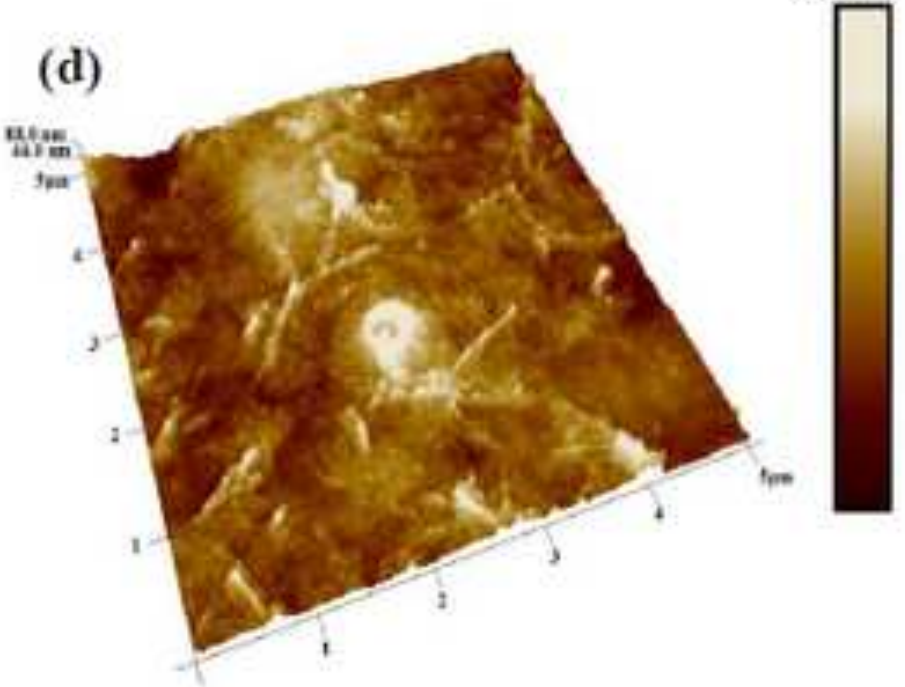

$\mathrm{R}_{\mathrm{a}}: 8.87 \mathrm{~nm}$

Surface area difference: $4.90 \%$ 


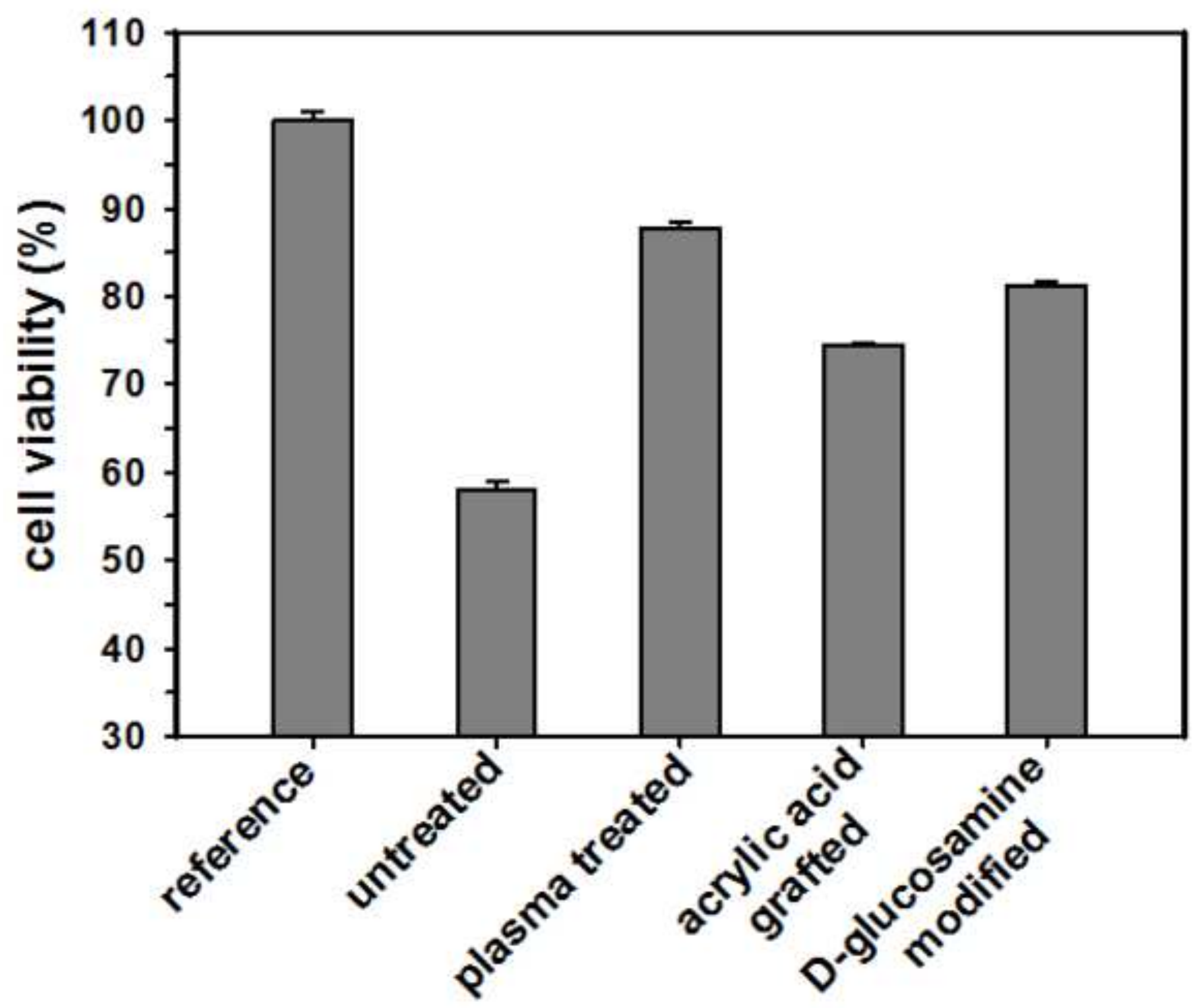



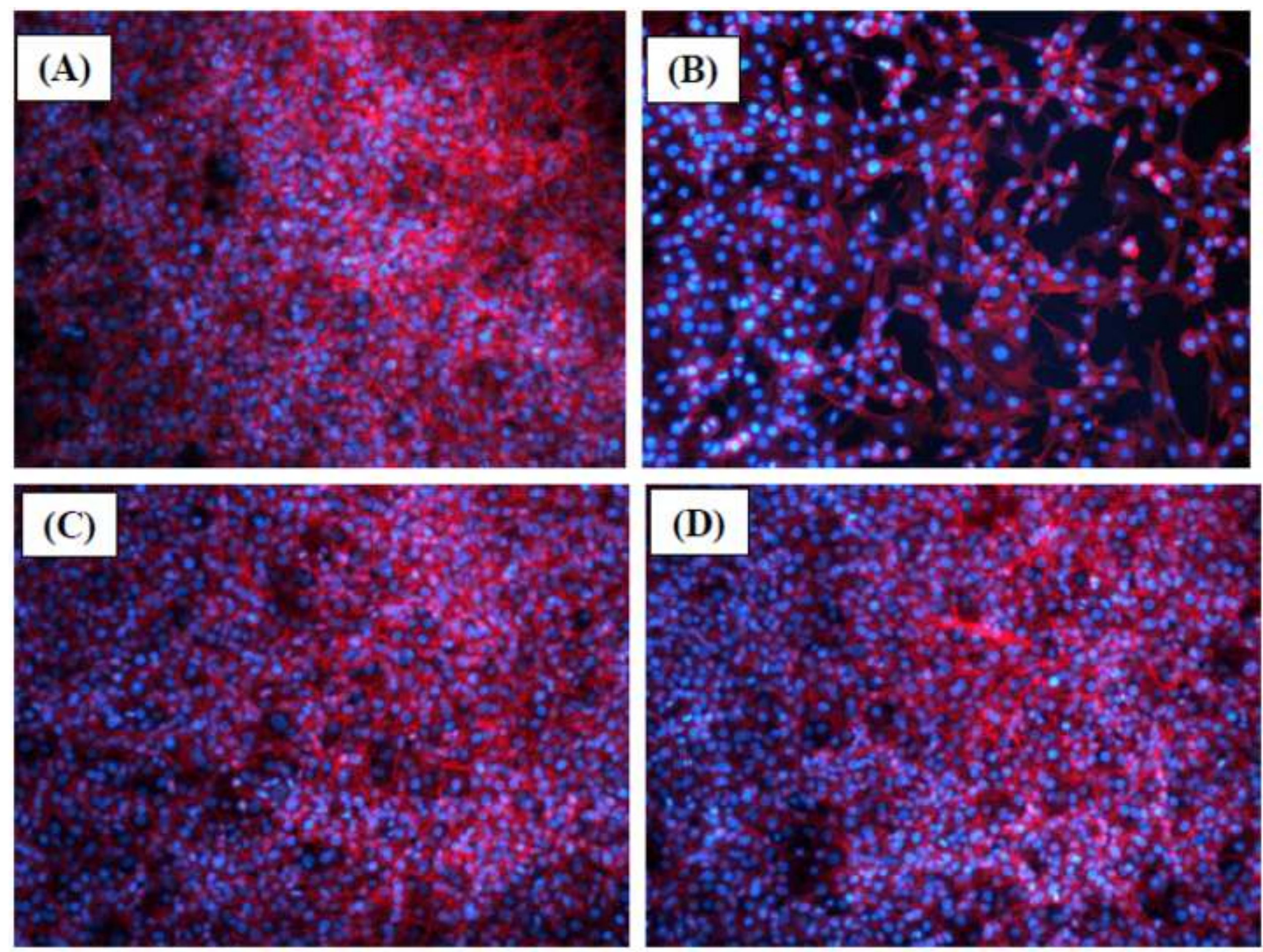